Journal of Universal Mathematics

Vol.4 No.2 PP.102-108 (2021)

ISSN-2618-5660

DOI:10.33773/jum.967903

\title{
WEAKLY LOCALLY ARTINIAN SUPPLEMENTED MODULES
}

\author{
BURCU NIŞANCI TÜRKMEN
}

0000-0001-7900-0529

\begin{abstract}
In this study, by using the concept of locally artinian supplemented modules, we have obtained weakly locally artinian supplemented modules as a proper generalization of these modules in module theory. Our results generalize and extend various comparable results in the existing literature. We have proved that the notion of weakly locally artinian supplemented modules inherited by factor modules, finite sums and small covers. We have obtained that weakly locally artinian supplemented modules with small radical coincide with weakly (radical) supplemented modules which have locally artinian radical. Also, we have shown that if $N$ and $\frac{M}{N}$ are weakly locally artinian supplemented for some submodule $N \subseteq M$ which has a weak locally artinian supplement in $M$ then $M$ is weakly locally artinian supplemented.
\end{abstract}

\section{INTRODUCTION}

Throughout this paper, the ring $R$ will denote an associative ring with identity element and modules will be left unital. We will use the notation $U \ll M$ to stress that $U$ is a small submodule of $M$. A submodule $N \subseteq M$ is said to be essential in M, denoted as $N \unlhd M$, if $N \cap K \neq 0$ for every non-zero submodule $K \subseteq M$. By $\operatorname{Rad}(M)$ we denote the sum of all small submodules of $M$ or, equivalently the intersection of all maximal submodules of $M$. $\operatorname{Soc}(M)$ will indicate socle of $M$ which is sum of all semisimple submodules of $M$. A non-zero module $M$ is called hollow if every proper submodule of $M$ is small in $M$, and $M$ is called local if the sum of all proper submodules of $M$ is also a proper submodule of $M$. A module $M$ is called semilocal if $\frac{M}{\operatorname{Rad}(M)}$ is semisimple. A ring $R$ is said to be semilocal if $\frac{R}{\operatorname{Rad}(R)}$ is semisimple. By [5, Proposition 20.2], a commutative ring $R$ is semilocal if and only if $R$ has only finitely many maximal ideals. $M$ is called locally artinian if every finitely generated submodule of $M$ is artinian [10,31]. A submodule $V$ of $M$ is called a supplement of $U$ in $M$ if $M=U+V$ and $U \cap V \ll V$. A submodule $V$ of $M$ is called $a$ weak supplement of $U$ in $M$ if $M=U+V$ and $U \cap V \ll M$. The module $M$ is called (weakly) supplemented if every submodule of $M$ has a (weak) supplement in $M$. In [1], it is proved that the class of weakly supplemented modules need not be

Date: Received: 2021-07-08; Accepted: 2021-07-29.

2000 Mathematics Subject Classification. 16D10, 16N20; 16D99.

Key words and phrases. Locally artinian module, (Weak) supplement, (Weak) locally artinian supplement, (Weakly) locally artinian supplemented module. 
closed under extensions, that is if $U$ and $M / U$ are weakly supplemented for some submodule $U$ of $M$ then $M$ need not be weakly supplemented. A submodule $U$ of $M$ has ample supplements in $M$ if every submodule $V$ of $M$ such that $M=U+V$ contains a supplement $V^{\prime}$ of $U$ in $M$. The module $M$ is called amply supplemented if every submodule of $M$ has ample supplements in $M$ [10].

Let $R$ be a a principal ideal domain (PID) with exactly one non-zero maximal ideal, then $R$ is said to be a Discrete valuation ring (DVR). By [13, Lemma 2.1] that every module with small radical over a Discrete Valuation Ring, is the direct sum of a finitely generated free module and a bounded module. In [12], he generalized the concept of modules with small radical to radical supplemented modules. $M$ is called radical supplemented if $\operatorname{Rad}(M)$ has a supplement in $M$. These modules are also a proper generalization of supplemented modules. Then, in [2], it is defined as a module $M$ strongly radical supplemented (or briefly srs) if every submodule $N$ of $M$ with $\operatorname{Rad}(M) \subseteq N$ has a supplement in $M$. Then it is introduced that modules whose every submodule containing the radical has a weak supplement (in particular, over dedekind domains the radical has a weak supplement) in the module as weakly radical supplemented module (wrs) which is a generalization of strongly radical supplemented modules [7].

In [11], a generalization of concept of socle as a $\operatorname{Soc}_{s}(M)=\sum\{U \ll M \mid U$ is simple $\}$. Here $\operatorname{Soc}_{s}(M) \subseteq \operatorname{Rad}(M)$ and $\operatorname{Soc}_{s}(M) \subseteq \operatorname{Soc}(M)$. In [3], a module $M$ is called strongly local if it is local and $\operatorname{Rad}(M)$ is semisimple. A submodule $U$ of $M$ is called an ss-supplement of $U$ in $M$ if $M=U+V$ and $U \cap V \subseteq \operatorname{Soc}_{s}(V)$. The module $M$ is called ss-supplemented if every submodule of $M$ has an $s s$-supplement in $M$. A submodule $U$ of $M$ has ample $s s$-supplements in $M$ if every submodule $V$ of $M$ such that $M=U+V$ contains an $s s$-supplement $V^{\prime}$ of $U$ in $M$. The module $M$ is called amply ss-supplemented if every submodule of $M$ has ample ss-supplements in $M$. In [8], strongly local and (amply) ss-supplemented modules are generalized as $R L A$-local and (amply) locally artinian supplemented modules, respectively. A local module $M$ is called $R L A$-local if $\operatorname{Rad}(M)$ is a locally artinian submodule of $M$. A module $M$ is called locally artinian supplemented if every submodule $U$ of $M$ has a locally artinian supplement in $M$, that is, $V$ is a supplement of $U$ in $M$ such that $U \cap V$ is locally artinian. $M$ is called amply locally artinian supplemented if every submodule $U$ of $M$ has ample locally artinian supplements in $M$. Here a submodule $U$ of $M$ has ample locally artinian supplements in $M$ if every submodule $V$ of $M$ such that $M=U+V$ contains a locally artinian supplement $V^{\prime}$ of $U$ in $M$.

In Section 2, it is proved that a module with a small radical is weakly locally artinian supplemented if and only if $M$ is weakly supplemented and $\operatorname{Rad}(M)$ is locally artinian. It is also proved that finite sum of weakly locally artinian supplemented modules is weakly locally artinian supplemented and every factor module of a weakly locally artinian supplemented module is weakly locally artinian supplemented. It is shown that a notion of weakly locally artinian supplemented modules inherited by small cover. It is also shown that if $N$ and $\frac{M}{N}$ are weakly locally artinian supplemented for some submodule $N \subseteq M$ which has a weak locally artinian supplement in $M$, then $M$ is weakly locally artinian supplemented. 


\section{Weakly Locally Artinian Supplemented Modules}

Definition 1. Let $M$ be a module. Then $M$ is called weakly locally artinian supplemented if every submodule $N$ of $M$ has a weak supplement $K$ in $M$ with $N \cap K$ is locally artinian, i.e. $N$ has a weak locally artinian supplement $K$ in $M$.

By the definition, it is clear that every weakly locally artinian supplemented module is weakly supplemented. The following example shows that the converse is not always true.

Example 1. Consider the $\mathbb{Z}$-module $\mathbb{Q}$. By [1, Lemma 2.8], $M=\mathbb{Z} \mathbb{Q}$ is weakly supplemented. So $\frac{\mathbb{Q}}{\mathbb{Z}}$ is weakly supplemented because of $\mathbb{Q}$ is weakly supplemented. But $\frac{\mathbb{Q}}{\mathbb{Z}}$ is not locally artinian by $\left[9\right.$, Theorem 3]. Since $\operatorname{Rad}\left(\frac{\mathbb{Q}}{\mathbb{Z}}\right)=\frac{\mathbb{Q}}{\mathbb{Z}}, \frac{\mathbb{Q}}{\mathbb{Z}}$ is not weakly locally artinian supplemented.

Lemma 1. Let $M$ be a weakly supplemented module and $\operatorname{Rad}(M)$ be a locally artinian submodule of $M$. Then $M$ is weakly locally artinian supplemented.

Proof. Let $N \subseteq M$. By the hypothesis, there exists a submodule $K$ of $M$ such that $M=N+K, N \cap K \ll M$. So $N \cap K \subseteq \operatorname{Rad}(M)$. Since $\operatorname{Rad}(M)$ is a locally artinian submodule of $M, N \cap K$ is a locally artinian submodule of $M$ by [10, 31.2 (ii)]. Thus $M$ is weakly locally artinian supplemented.

Theorem 1. Let $M$ be a module with small radical. Then the following statements are equivalent.

(1) $M$ is weakly locally artinian supplemented;

(2) $M$ is weakly supplemented and $\operatorname{Rad}(M)$ has a weak locally artinian supplement in $M$;

(3) $M$ is weakly supplemented and $\operatorname{Rad}(M)$ is locally artinian.

Proof. (1) $\Rightarrow(2)$ Since $M$ is weakly locally artinian supplemented, $M$ is weakly supplemented and $\operatorname{Rad}(M)$ has a weak locally artinian supplement in $M$.

$(2) \Rightarrow(3)$ Since $\operatorname{Rad}(M) \ll M, M$ is a weak locally artinian supplement of $\operatorname{Rad}(M)$ in $M$. Thus we have $M=\operatorname{Rad}(M)+M, \operatorname{Rad}(M)=\operatorname{Rad}(M) \cap M \ll M$ and $\operatorname{Rad}(M)$ is locally artinian.

$(3) \Rightarrow(1)$ Clear by Lemma 1.

Since every finitely generated module has a small radical, we have:

Corollary 1. Let $M$ be a finitely generated module. Then $M$ is weakly locally artinian supplemented if and only if $M$ is weakly supplemented with locally artinian radical.

Proposition 1. Let $M$ be a weakly locally artinian supplemented module and $N, K \subseteq M$ be submodules with $M=N+K$. Then $K$ contains a weak locally artinian supplement $K^{\prime}$ of $N$ in $M$.

Proof. Let $N \cap K=U$. Since $M$ is weakly locally artinian supplemented, there exists a submodule $V$ of $M$ such that $M=U+V, U \cap V \ll M$ and $U \cap V$ is locally artinian. Then $K=K \cap(U+V)=U+(K \cap V)$ and $M=N+K=$ $N+[U+(K \cap V)]=N+(K \cap V)$. It follows that $N \cap(K \cap V)=U \cap V \ll M$ and $U \cap V$ is locally artinian, say $K^{\prime}=K \cap V$. Then we obtain that $K^{\prime}$ is a weak locally artinian supplement of $N$ in $M$, as required. 
Proposition 2. Let $M$ be a weakly locally artinian supplemented module and $N$ be a small submodule of $M$. Then $N$ is locally artinian.

Proof. By the hypothesis, there exists a submodule $K$ of $M$ such that $M=N+K$, $N \cap K \ll M$ and $N \cap K$ is locally artinian. Since $N \ll M, M=K$. So $N \cap K=$ $N \cap M=N$ is locally artinian.

Corollary 2. Let $M$ be a weakly locally artinian supplemented module and $\operatorname{Rad}(M)$ $\ll M$. Then $\operatorname{Rad}(M)$ is locally artinian.

Proof. Clear by Proposition 2.

With the help of the next theorem, we verify that under special conditions, notions of weakly locally artinian supplemented modules and weakly radical supplemented modules are the same.

Theorem 2. Let $M$ be a module with $\operatorname{Rad}(M) \ll M$. Then the following statements are equivalent.

(1) $M$ is weakly locally artinian supplemented;

(2) $M$ is weakly supplemented and Rad(M) has a weak locally artinian supplement in $M$;

(3) $M$ is weakly supplemented and $\operatorname{Rad}(M)$ is locally artinian;

(4) $M$ is weakly radical supplemented and $\operatorname{Rad}(M)$ is locally artinian.

Proof. (1) $\Leftrightarrow(2) \Leftrightarrow(3)$ Clear by Theorem 1 .

(3) $\Rightarrow$ (4) Obvious.

(4) $\Rightarrow(1)$ Let $N \subseteq M$. By the hypothesis, $N+\operatorname{Rad}(M)$ has a weak supplement $K$ in $M$. Then we have $M=(N+\operatorname{Rad}(M))+K$. Since $\operatorname{Rad}(M) \ll M, M=N+K$, $N \cap K \subseteq(N+\operatorname{Rad}(M)) \cap K \ll M$. So $N \cap K \ll M$. Thus $N \cap K \subseteq \operatorname{Rad}(M)$. Since $\operatorname{Rad}(M)$ is locally artinian, $N \cap K$ is locally artinian by [10, 31.2 (ii)]. Therefore $K$ is a weak locally artinian supplement of $N$ in $M$, as desired.

We will show that in the factor modules, the property is preserved in weakly locally artinian supplemented modules just as it is in weakly supplemented modules.

Proposition 3. If $M$ is a weakly locally artinian supplemented module, then every factor module of $M$ is weakly locally artinian supplemented.

Proof. Let $M$ be a weakly locally artinian supplemented module and $\frac{M}{N}$ be a factor module of $M$. By the assumption, for any submodule $N \subseteq U \subseteq M$, there exists a submodule $V$ of $M$ such that $M=U+V, U \cap V \ll M$ and $U \cap V$ is locally artinian. Let $\pi: M \longrightarrow \frac{M}{N}$ be the canonical projection. Then we have $\frac{M}{N}=\frac{U}{N}+\frac{V+N}{N}$, $\frac{U}{N} \cap \frac{V+N}{N}=\pi(U \cap V) \ll \frac{M}{N}$ and $\frac{U}{N} \cap \frac{V+N}{N}=\pi(U \cap V)$ is locally artinian by [10, $31.2(\mathrm{i})]$, as required.

The following lemma plays a key role in showing that the notion of weakly locally artinian supplemented modules is inherited by finite sum.

Lemma 2. Let $M$ be a module, $M_{1} \subseteq M, N \subseteq M$ and $M_{1}$ be weakly locally artinian supplemented. If $M_{1}+N$ has a weak locally artinian supplement in $M$, then $N$ has a weak locally artinian supplement in $M$.

Proof. Let $K$ be a weak locally artinian supplement of $M_{1}+N$ in $M$. Then we can write $M=\left(M_{1}+N\right)+K,\left(M_{1}+N\right) \cap K \ll M$ and $\left(M_{1}+N\right) \cap K$ is locally artinian. 
Since $M_{1}$ is weakly locally artinian supplemented, $(N+K) \cap M_{1}$ has a weak locally artinian supplement $L$ in $M_{1}$, i.e. $M_{1}=(N+K) \cap M_{1}+L,(N+K) \cap L \ll M_{1}$ and $(N+K) \cap L$ is locally artinian. Then $M=M_{1}+(N+K)=\left[(N+K) \cap M_{1}+\right.$ $L]+(N+K)=N+(K+L)$ and $N \cap(K+L) \subseteq K \cap(N+L)+L \cap(N+K) \subseteq$ $K \cap\left(N+M_{1}\right)+L \cap(N+K) \ll M$. By [10, 31 (2) (i), (ii)], $N \cap(K+L)$ is locally artinian and so $K+L$ is a weak locally artinian supplement of $N$ in $M$.

Corollary 3. Let $M$ be an $R$-module, $N \subseteq M$ and $M_{i} \subseteq M$ for $i=1,2, \ldots, n$. If $N+M_{1}+\cdots+M_{n}$ has a weak locally artinian supplement in $M$ and $M_{i}$ is a weakly locally artinian supplemented module for every $i=1,2, \ldots, n$, then $N$ has a weak locally artinian supplement in $M$.

Corollary 4. Let $M=M_{1}+M_{2}$. If $M_{1}$ and $M_{2}$ are weakly locally artinian supplemented modules, then $M$ is a weakly locally artinian supplemented module.

The following corollary is obtained from the previous result by applying induction.

Corollary 5. A finite sum of weakly locally artinian supplemented modules is weakly locally artinian supplemented.

Recall from [6] that $N$ is a small cover of a module $M$ if there exists an epimorphism $f: N \longrightarrow M$ such that $\operatorname{Ker}(f) \ll M$.

Lemma 3. Let $M$ be a weakly locally artinian supplemented module. Then every small cover of $M$ is weakly locally artinian supplemented.

Proof. Let $N$ be a small cover of $M$. Then there exists an epimorphism $f: N \longrightarrow M$ such that $\operatorname{Ker}(f) \ll N$. Note that $f^{-1}(K) \ll N$ for every $K \ll M$ holds since $\operatorname{Ker}(f) \ll N$. Let $L \subset N$. Then $f(L)$ has a weak locally artinian supplement of $X$ in $M$. Note that $M=X+f(L), X \cap f(L) \ll M$ and $X \cap f(L)$ is locally artinian. Again it is easy to check that $f^{-1}(X)$ is a weak locally artinian supplement of $L$ in $N$.

Proposition 4. Let $M$ be a weakly locally artinian supplemented module. Then every locally artinian supplement in $M$ is weakly locally artinian supplemented.

Proof. Let $K$ be a locally artinian supplement of $N$ in $M$. Then we have $M=$ $N+K, N \cap K \ll K$ and $N \cap K$ is locally artinian. $\frac{M}{N} \cong \frac{K}{(N \cap K)}$ is weakly locally artinian supplemented by Proposition 3 . By Lemma $3, K$ is weakly locally artinian supplemented.

Corollary 6. Let $M$ be a weakly locally artinian supplemented module. Then every locally artinian direct summand in $M$ is weakly locally artinian supplemented.

Proof. Since every locally artinian direct summand is locally artinian supplement, the proof follows from Proposition 4.

Recall that a submodule $N \subseteq M$ is called closed in $M$ if $N \unlhd K$ for some $K \subseteq M$ implies $K=N$. A submodule $N \subseteq M$ is called coclosed in $M$ if $\frac{N}{K} \ll \frac{M}{K}$ for some $K \subseteq M$ implies $K=N$.

Theorem 3. Let $0 \longrightarrow K \longrightarrow M \longrightarrow N \longrightarrow 0$ be a short exact sequence. If $K$ and $N$ are weakly locally artinian supplemented and $K$ has a weak locally artinian supplement in $M$ then $M$ is weakly locally artinian supplemented. If $K$ 
is coclosed locally artinian submodule in $M$ then the converse holds, that is if $M$ is weakly locally artinian supplemented then $K$ and $N$ are weakly locally artinian supplemented.

Proof. Without restriction of generality we will assume that $K \subseteq M$. Let $T$ be a weak locally artinian supplement of $K$ in $M$ i.e. $M=K+T, K \cap T \ll M$ and $K \cap T$ is locally artinian. Then we have, $\frac{M}{(K \cap T)}=\frac{K}{(K \cap T)} \oplus \frac{T}{(K \cap T)}$. Since $\frac{K}{(K \cap T)}$ is a factor module of $K, \frac{K}{(K \cap T)}$ is weakly locally artinian supplemented by Proposition 3. On the other hand, $\frac{T}{(K \cap T)} \cong \frac{M}{K} \cong N$ is weakly locally artinian supplemented by the hypothesis. Then, by Corollary $5, \frac{M}{(K \cap T)}$ is weakly locally artinian supplemented as a finite sum of weakly locally artinian supplemented modules. It follows from Lemma 3 that $M$ is weakly locally artinian supplemented.

Suppose that $M$ is weakly locally-artinian supplemented and $K$ is a coclosed locally-artinian submodule in $M$. Then $K \cap T \ll K$ by [4, Lemma 1.1] and $K \cap T$ is locally artinian by $[10,31.2$ (ii)] i.e. $K$ is a locally artinian supplement of $T$ in $M$. Therefore $K$ is weakly locally artinian supplemented by Proposition 4 .

Recall from [6, Theorem 3.5] that a ring $R$ is semilocal if and only if every $R$ module with small radical is weakly supplemented. By using Theorem 1, we have the following Proposition.

Proposition 5. Let $R$ be a semilocal ring and $M$ be an $R$-module. Suppose $N \subseteq M$ such that $\frac{M}{N}$ is finitely generated and $\operatorname{Rad}\left(\frac{M}{N}\right)$ is locally artinian. If $N$ is weakly locally artinian supplemented then $M$ is weakly locally artinian supplemented.

Proof. Suppose $\frac{M}{N}$ is generated by $m_{1}+N, m_{2}+N, \ldots, m_{n}+N$. For the submodule $K=R m_{1}+R m_{2}+\cdots+R m_{n}$, we have $M=N+K$. Then $M$ is weakly locally artinian supplemented by Corollary 4 .

\section{Conclusion}

The aim of this paper is to reveal the existence of the concept of weakly locally artinian supplemented modules. Our results improve and generalize some known results on locally artinian supplemented modules.

\section{Acknowledgments}

The authors would like to thank the reviewers and editors of Journal of Universal Mathematics.

\section{Funding}

The author(s) declared that has no received any financial support for the research, authorship or publication of this study.

\section{The Declaration of Conflict of Interest/ Common Interest}

The author(s) declared that no conflict of interest or common interest

\section{The Declaration of Ethics Committee Approval}

This study does not be necessary ethical committee permission or any special permission. 


\section{The Declaration of Research and Publication Ethics}

The author(s) declared that they comply with the scientific, ethical, and citation rules of Journal of Universal Mathematics in all processes of the study and that they do not make any falsification on the data collected. Besides, the author(s) declared that Journal of Universal Mathematics and its editorial board have no responsibility for any ethical violations that may be encountered and this study has not been evaluated in any academic publication environment other than Journal of Universal Mathematics.

\section{REFERENCES}

[1] R. Alizade, E. Büyükaşık. Extensions of weakly supplemented modules, Math. Scand., Vol. 103, pp. 161-168 (2008).

[2] E. Büyükaşık, E. Türkmen. Strongly radical supplemented modules. Ukr. Math. J., 63, No. 8, 1306-1313 (2011).

[3] E. Kaynar, H. Çalışıcı, E. Türkmen. ss-supplemented modules. Communications Faculty of Science University of Ankara Series A1 Mathematics and statistics, Vol. 69, 1, pp 473-485 (2020).

[4] D. Keskin. On lifting modules, Comm. Algebra, Vol.28:7, 3427-3440 (2000).

[5] T.Y. Lam. A first course in noncommutative rings, Springer, New York (1999).

[6] C. Lomp. On semilocal modules and rings, Comm. Algebra, Vol. 27:4, 1921-1935 (1999).

[7] B. Nişancı Türkmen, E. Türkmen. On a generalization of weakly supplemented modules. An. Stiin. Univ. Al. I. Cuza Din Iasi. Math (N.S.), Vol. 63(2), pp. 441-448 (2017).

[8] Y. Şahin, B. Nişancı Türkmen. Locally-artinian supplemented modules. 9th International Eurasian Conference On Mathematical Sciences and Applications Abstract Book,Skopje, North Macedonia, pp. 26 (2020).

[9] D. Van Huynh, R. Wisbauer. Characterization of locally artinian modules. Journal of Algebra, Vol. 132, pp. 287-293 (1990)

[10] R. Wisbauer. Foundations of modules and rings. Gordon and Breach, Springer-Verlag (1991).

[11] D. X. Zhou, X. R. Zhang. Small-essential submodules and morita duality. Southeast Asian Bulletin of Mathematics, Vol. 35, pp 1051-1062 (2011).

[12] H. Zöschinger. Moduln, die in jeder erweiterung ein komplement haben. Math. Scand., Vol. 35 , pp. 267-287 (1974).

[13] H. Zöschinger. Komplementierte moduln über dedekindringen. Journal of Algebra, Vol. 29, pp. $42-56$ (1974).

(Burcu Nişancı Türkmen) Amasya university, Mathematics department, 05100, Amasya, TURKEY

Email address, Burcu Nişancı Türkmen: burcu.turkmen@amasya.edu.tr 